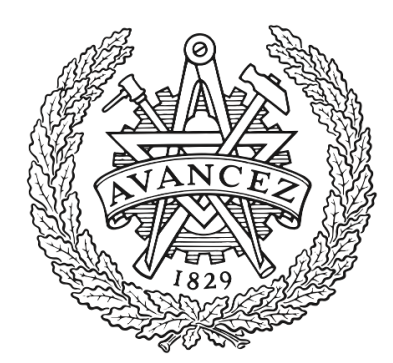

CHALMERS

\title{
Faulted Feeder Identification Based on Active Adjustment of Arc Suppression Coil and Similarity Measure of Zero-Sequence Currents
}

Downloaded from: https://research.chalmers.se, 2023-04-26 14:59 UTC

Citation for the original published paper (version of record):

Tang, J., Xiong, B., Li, Y. et al (2021). Faulted Feeder Identification Based on Active Adjustment of Arc Suppression Coil and Similarity

Measure of Zero-Sequence Currents. IEEE Transactions on Power Delivery, 36(6): 3903-3913.

http://dx.doi.org/10.1109/TPWRD.2021.3051040

N.B. When citing this work, cite the original published paper.

(C)2021 IEEE. Personal use of this material is permitted.

However, permission to reprint/republish this material for advertising or promotional purposes 


\title{
Faulted Feeder Identification Based on Active Adjustment of Arc Suppression Coil and Similarity Measure of Zero-Sequence Currents
}

\author{
Jinrui Tang, Member, IEEE, Binyu Xiong, Member, IEEE, Yang Li, Member, IEEE, Chengqing Yuan, \\ and Yuanchao Qiu
}

\begin{abstract}
Existing faulted feeder identification methods in the resonant grounded distribution network are primarily based on feature extraction of the fault-generated transient currents. The reliability of these approaches is significantly compromised by the fluctuating transient signals and interfering on-off operation of the neighboring switches. To sidestep the problems, a novel method is proposed to identify the faulted feeder by consecutively tuning the arc suppression coil around the full compensation state. Once a series of steady states are reached after tuning, the trajectories of the corresponding zero-sequence currents for both the sound and the faulted feeders are obtained to formulate an adjustment trajectory matrix (ATM). With the ATM, the similarity measure of the adjustment trajectories of all feeders is then employed to identify the faulted feeder based on the selected Deng's grey relational analysis. Results show that the adjustment trajectories of the two sound lines share a high similarity degree, while the similarity between the sound and the faulted lines is much lower. The effectiveness of the proposed method is validated via simulation and some case studies are provided. The results show that the faulted feeder can be correctly identified with high reliability and robustness compared to the existing fault-generated signal-based techniques.
\end{abstract}

Index Terms - arc suppression coil (ASC), faulted feeder identification, grey relational analysis (GRA), similarity measure, zero-sequence current (ZSC)

\section{INTRODUCTION}

$\mathrm{D}$ UE to the recent fast expansion of the distribution network and wide application of underground cables in China, the magnitude of the capacitive earth-fault currents increased significantly, and it leads to severe single-phase-to-ground (SPG) faults. Such high-current faults can cause damage to sophisticated electrical devices and pose hazards to the people nearby [1]. One common method to alleviate the impact of the SPG faults is to introduce arc suppression coils (ASCs) for fault current suppression [2], resulting in an ASC-grounded resonant grounded distribution network (RGDN). By using ASCs, an RGDN is allowed to operate under a fault condition for $1-2$ hours before the fault is cleared [3]. However, this also brings great challenges for faulted feeder identification, since the

This work was supported in part by the National Natural Science Foundation of China under Grant 51707139.

Jinrui Tang, Binyu Xiong, and Yang Li are with the School of Automation, Wuhan University of Technology, Wuhan 430070, China (e-mail: tangjinrui@whut.edu.cn; bxiong2@whut.edu.cn; yangli@ieee.org).

Chengqing Yuan and Yuanchao Qiu are with the School of Energy and Power Engineering, Wuhan University of Technology, Wuhan 430070, China (e-mail: ycq@whut.edu.cn; qycwhut@163.com). suppressed residual fault current is too low to distinguish amongst the sound and faulted lines after the compensation [4].

Existing faulted feeder identification methods in the RGDNs can be generally categorized into two groups. First, the fault-generated signal-based methods, which are deemed to be the traditional faulted feeder identification methods, extract the features from transient zero-sequence current (ZSC) signals within a short period (usually $100 \mathrm{~ms}$ ) after SPG fault occurs [5-12]. A typical procedure is to process the measured signals in the time or frequency domain and then analyze the features, such as the magnitude or the phase variation, for fault identification. In [5], discrete wavelet transform (DWT) was proposed to extract the features of transient signal spectrum beyond $6 \mathrm{kHz}$ within $40 \mathrm{~ms}$ after the fault, and a fault identification criterion is then set up based on probability method. Adaptive methods such as empirical mode decomposition [6] and mathematical morphology [7] were proposed to overcome the difficulty of mother wavelet selection in DWT. Another challenge for fault identification in RGDNs is that the transient signal profiles may vary significantly since they are largely dependent on factors including network topology, line parameters, and fault inception angle conditions. To improve the robustness of the algorithms under random fault signals profiles, theoretical studies on time-domain transient current characteristics of SPG were investigated [8]. A concept, namely, specific frequency spectrum band, was proposed in [9] and such spectrum band was applied for current feature extraction. Furthermore, novel methods that combine time and frequency analysis [10], support vector machine [11], and artificial neural network [12] were utilized to extract the multiple features. The above methods are straightforward and based on the analysis of fault generated signals. Nevertheless, as the fault generated signals are transient, non-stationary, and fluctuated, they can be easily interfered by measured or operational noises, and it eventually leads to incorrect identification results or identification failure.

Second, the auxiliary devices generated signal-based methods, which are considered to be the emerging faulted feeder identification methods, use external sources to generate active disturbance signals for fault identification. It was observed that the impedance of auxiliary devices, such as the controllable resistive circuit at the neutral point [13], signal generator at the secondary opening delta winding of potential transformer (PT) [14], and controllable reactors of suppression coils [15-18], would vary after the SPG fault occurs. 
Furthermore, the equivalent zero-sequence impedance of the distribution system would also vary in response to such variation, and a dynamic signal can be captured and utilized for fault identification. In [13], two thyristor-controlled grounding devices were proposed to adjust the grounding resistance by injecting a transient current pulse to the distribution system, and the injected signals can only be detected in the faulted feeder. To mitigate the impacts on the excitation circuit of the step-up transformer when the thyristors are at the on-state, a signal generator was installed at the secondary opening delta winding of the PT [14]. The methods in this category demonstrate high reliability, since the impedance of auxiliary devices is controllable, and the fault feature is much more identifiable compared with the previous group of methods.

As the most widely used grounding device in RGDNs, an ASC can be adopted as an auxiliary device due to its self-tuning ability for arc suppression. In [15], the impedance of the ASC was tuned around the full compensation state after the SPG fault occurs. After a certain time (usually about $100 \mathrm{~ms}$ ), the ASC was adjusted to the overcompensation or under-compensation state, and meanwhile, the corresponding variations of the feeder currents were measured. The feeder with the greatest ZSC variation was identified as the faulted line. However, further study showed that the above method may not be reliable especially in a system with high fault transition resistance, since the signal variations of the sound feeders may be even greater than that of the faulted one [16]. To enhance the reliability, a criterion that combines the variations in transient current and transient voltage was proposed in [17]. Similarly, in [18], the authors introduced the approach to use the features of steady-state current and voltage variations for fault identification. These two methods show high reliability, but additional devices have to be installed and extra cost and reconstruction work is needed. In [19], the relationships between the steady-state ZSC variation, line parameters, and compensation degree were analyzed in detail. In addition, recent works have shown that similarity analysis could be a potential method for fault identification [20-22]. In [20], the waveform similarity between the captured differential current sequence and a standard sine wave sequence is calculated, and the result was used to identify the fault types of the power transformer based on Hausdorff-distance (HD) algorithm. The HD algorithm was also adopted to obtain the similarity of the currents to establish the protection criterion for the transmission lines in [21]. In [22], the similarity analysis of the fault-generated transient current signals based on grey relational analysis (GRA) was first proposed for the identification of the faulted feeder in the RGDNs. The theoretical analysis and simulation results show that such a transient waveform similarity-based algorithm is capable of detecting the faulted feeder accurately in the RGDNs. Unfortunately, the reliability of this method is again limited by the transient, non-stationary, and easily-interfered fault-generated signals.

It can be seen that traditional faulted feeder identification methods mainly use transient ZSC signals to extract features for identification. Due to the non-stationary and fluctuating nature of the transient process, incorrect identification or identification failure occurs regularly in the field. Owing to the excellent adjustment ability of ASC, the effectiveness of auxiliary devices generated signal-based methods has recently been proven. Existing ASC generated signal-based methods usually use the variations of ZSCs as features to identify the faulted feeder [15-18]. Although these features can avoid identification failure, incorrect detection results are usually obtained since the variation features of ZSCs are irregular and implicit, especially at high fault resistance and weak signals.

In order to sidestep the above-mentioned problems, in this paper, a novelty method is proposed by consecutively tuning the ASC so that a series of steady states are obtained. The faulted feeder identification problem is converted to find the similarity difference of current adjustment trajectories with a constructed adjustment trajectory matrix (ATM). Each element in the ATM is extracted from the steady-state current by adjusting the ASC under different compensation degrees. Meanwhile, Deng's GRA [23] is selected as the appropriate similarity measurement method among different types of similarity measurements, such as the cosine similarity and HD algorithms used in [20-22]. The unique characteristics of Deng's GRA are that it requires a small number of matrix elements, which can reduce the adjusting times for the ASC and improve antijamming capability. Another merit of Deng's GRA is that the robustness of the algorithm is guaranteed even when the fault resistance or the compensation degree varies in a wide range.

The rest of this paper is organized as follows. In Section II, the ATM of the ZSC during the active and multiple off-tuning adjustments of ASC is presented under the SPG fault. In Section III, a novel faulted feeder identification method by measuring the similarity of the row vectors in the ATM is proposed and illustrated. In Section IV, the reliability of the proposed method is verified experimentally. The robustness of the proposed method is presented and discussed in Section V. The concluding remarks are given in Section VI.

\section{Adjustment Trajectory Matrix OF Steady-State ZERO-SEQUENCE CURRENTS OF FEEDERS}

\section{A. Preliminaries}

The schematic diagram of a typical RGDN under present investigation is shown in Fig. 1. The ASC is accessible via a grounding transformer connected to the substation bus. An ASC regulator is used to adjust the inductance of the ASC by controlling the on-load tap changer according to a built-in arc-suppression algorithm. The arc-suppression algorithm usually uses the information on the recorded ZSCs flowing through all the feeders as well as the current flowing through the ASC.

Assume that an earth fault occurs on the phase A of Feeder $n$. The corresponding ZSC $\dot{I}_{0 i}$ of the $i$-th sound feeder and the ZSC $\dot{I}_{0 n}$ of the $n$-th faulted feeder can be expressed as, 


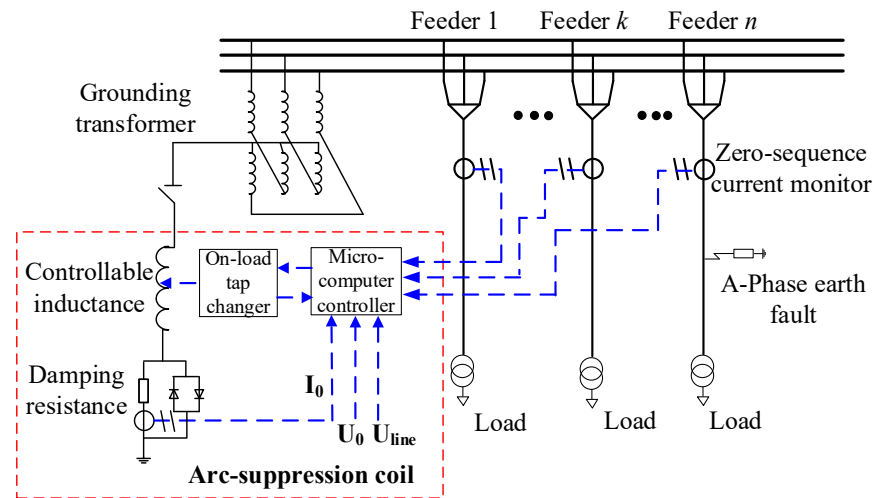

Fig. 1. Schematic diagram of a typical RGDN with a single-phase earth fault.

$$
\begin{aligned}
& \dot{I}_{0 i}=-\frac{\dot{E}_{A} j \omega C_{i}}{1-j p \times 3 \omega C R_{f}} \\
& \dot{I}_{0 n}=-\frac{\dot{E}_{A} j \omega\left(p C+C_{n}\right)}{1-j p \times 3 \omega C R_{f}}
\end{aligned}
$$

where $C_{i}$ and $C_{n}$ are the shunt capacitances of the $i$-th sound feeder and the $n$-th faulted feeder, respectively. $\dot{E}_{A}$ is the line-to-neutral voltage of the faulted phase (phase A), $R_{f}$ represents the single-phase earth fault transition resistance, $\omega$ is the synchronous angular speed, and $C$ is the total capacitance of all the feeders connected to the substation bus, i.e., $C=\sum_{i=1}^{n} C_{i}$. In traditional distribution networks, the compensation degree of the ASC is used to evaluate the arc suppression ability. The compensation degree $p$ is here defined as,

$$
p=\frac{\left|\dot{I}_{L}\right|-\left|\dot{I}_{C}\right|}{\left|\dot{I}_{C}\right|}=\frac{\left|\dot{U}_{g 0} / j 3 \omega L\right|-\left|\dot{U}_{g 0} j \omega C\right|}{\left|\dot{U}_{g 0} j \omega C\right|}=\frac{1 / 3 \omega L-\omega C}{\omega C}
$$

where $\dot{I}_{L}$ is the steady-state current flowing through the ASC, $\dot{I}_{C}$ is the total line-to-ground capacitive current of all the feeders connected to the bus, $\dot{U}_{g 0}$ is the neutral point voltage, and $L$ represents the inductance of the ASC.

Equations (1)-(3) will be used next to analyze the ATM of the steady-state ZSCs in the sound and the faulted feeders during the tuning of the ASC. In this paper, the ATM is defined as the combination of adjustment trajectories of all feeders. The adjustment trajectory of each feeder is represented by the magnitude sequences of power-frequency components of the ZSCs under different compensation degrees.

\section{B. Adjustment Trajectory Matrix of Steady-State Zero- Sequence Currents under Different Compensation Degrees}

The ZSC is influenced by the fault resistance and the compensation degree. The ZSCs of all feeders are monitored and recorded under the adjustments of ASC. The magnitudes of power-frequency components of the ZSCs in the sound and faulted feeders are extracted from these raw data based on Fourier transformation. After several adjustments (at least twice), the adjustment trajectories are stored to formulate the ATM. Specifically, the element in the adjustment trajectories, represented by $\dot{I}_{0 i}$ of the sound line and $\dot{I}_{0 n}$ of the faulted line, can be obtained by using (1) and (2). The adjustment trajectories of the sound feeder $i$, the sound feeder $k$, and the faulted feeder $n$ are shown in Fig. 2 as functions of $p$ at different $R_{f}$ values. From (3), it can be observed that the compensation degree can be adjusted accordingly by tuning the inductance of ASC. The adjustment trajectories of ZSCs of the faulted and any sound feeders under different SPG fault conditions are shown in Fig. 2. Note that the range of the compensation degree under consideration is $[-1.0,1.0]$. The operating states can be divided into three areas, i.e., the unrecommended area $[-1.0$, $-0.2]$, the operation area $[-0.2,0.2]$, and the infeasible area $[0.2$, $1.0]$. In practice, the system can only operate in the operation area $[-0.2,0.2]$ due to the constraints of arc suppression requirement, limited ASC adjusting ability, and the expansion of the distribution network. The adjustment trajectories presented in Fig. 2 are described by the following.

\section{1) Adjustment trajectory of ZSC $\dot{I}_{0 i}$ of the sound feeder $i$}

When an SPG fault with zero transition resistance $\left(R_{f}=0 \Omega\right)$ occurs, $\left|\dot{I}_{0 i}\right|$ is independent of the compensation degree $p$, and it remains constant when $p$ varies within a certain range. However, when $R_{f}$ increases, $\left|\dot{I}_{0 i}\right|$ would vary significantly with p. As shown in Fig. 2(a) to Fig. 2(f), the $\left|\dot{I}_{0 i}\right|$ vs. $p$ curve is symmetrical about the $p=0$ line. In addition, $\left|\dot{I}_{0 i}\right|$ decreases in either direction away from $p=0$. Furthermore, as $R_{f}$ increases, the rate of change of $\left|\dot{I}_{0 i}\right|$ with respect to $p$ increases drastically. In the meantime, the adjustment trajectories of the two sound lines, i.e., Feeder $i$ and Feeder $k$, have a high degree of similarity.

\section{2) Adjustment trajectory of ZSC $\dot{I}_{0 n}$ of the faulted feeder $n$}

Clearly, $\left|\dot{I}_{0 n}\right|$ is influenced by the transition resistance $R_{f}$ and the capacitance ratio $C_{n} / C$. When an SPG fault with zero transition resistance $\left(R_{f}=0 \Omega\right)$ occurs and $p=-C_{n} / C,\left|\dot{I}_{0 n}\right|=0$. In this condition, $\left|\dot{I}_{0 n}\right|$ is symmetrical about the $p=-C_{n} / C$ line. If an SPG fault occurs with non-zero transition resistance, it can be seen from Fig. 2 that the $\left|\dot{I}_{0 n}\right|$ vs. $p$ curves have nonlinear characteristics and they are asymmetrical about $p=-C_{n} / C$ line.

Figs. 2(a)-(c) show the adjustment trajectories of $\left|\dot{I}_{0 n}\right|$ for the faulted feeder with low $\left|C_{n} / C\right|$ under different fault resistances $R_{f}$, while Figs. 2(d)-(f) show the adjustment trajectories of $\left|\dot{I}_{0 n}\right|$ for the faulted feeder with high $\left|C_{n} / C\right|$. When $p$ varies from $-C_{n} / C$ to -1.0 , the adjustment trajectory of $\left|\dot{I}_{0 n}\right|$ increases with the decrease of $p$ under all fault conditions. On the other hand, when $p$ varies from 0 to $-C_{n} / C$, the adjustment trajectory of $\left|\dot{I}_{0 n}\right|$ decreases with the decrease of $p$. In the meantime, the variation trend of adjustment trajectory of $\left|\dot{I}_{0 n}\right|$ in the over-compensation region is directly determined by the fault resistance $R_{f}$. 


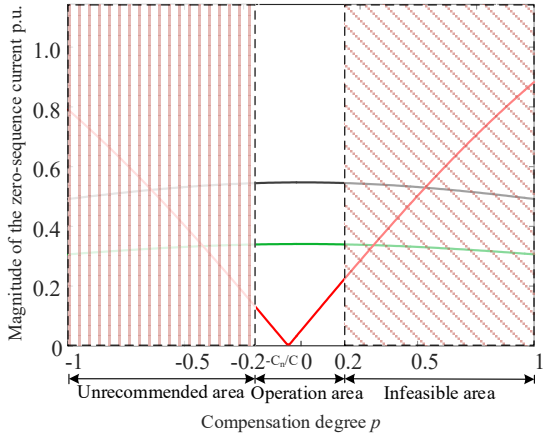

(a)

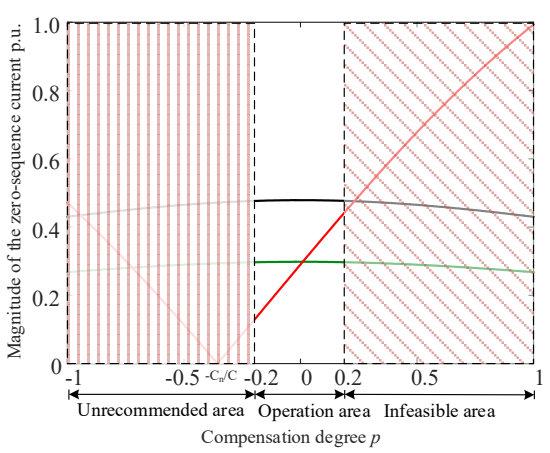

(d)

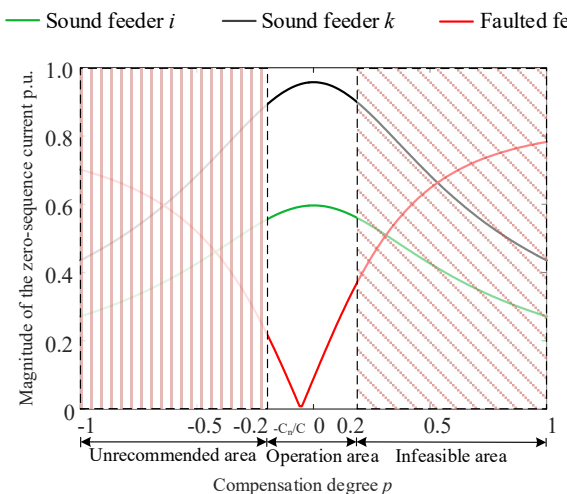

(b)

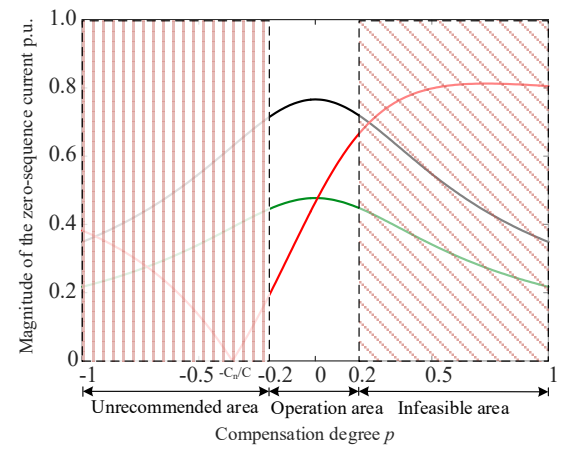

(e)

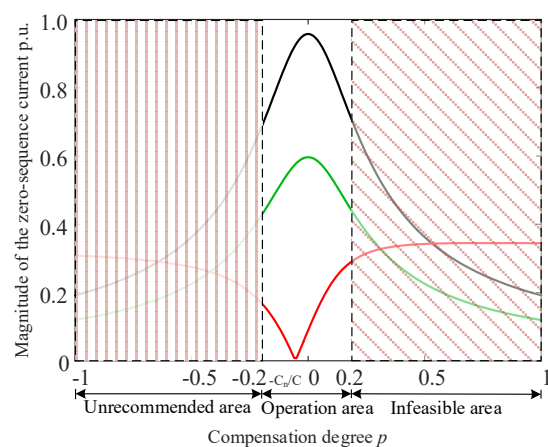

(c)

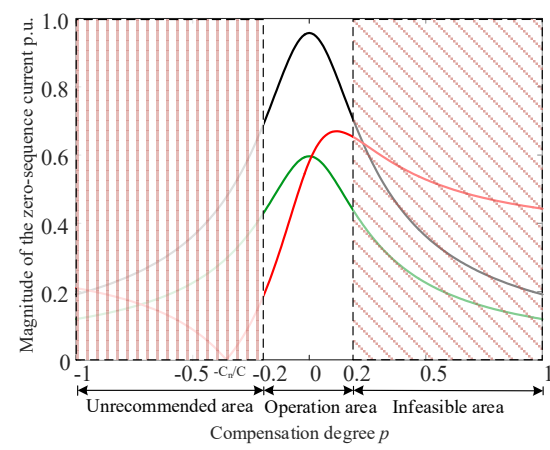

(f)

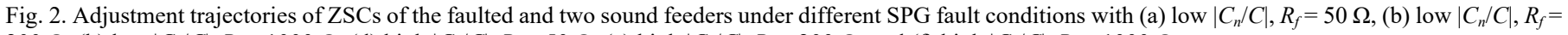
$200 \Omega$, (b) low $\left|C_{n} / C\right|, R_{f}=1000 \Omega$, (d) high $\left|C_{n} / C\right|, R_{f}=50 \Omega$, (e) high $\left|C_{n} / C\right|, R_{f}=200 \Omega$, and (f) high $\left|C_{n} / C\right|, R_{f}=1000 \Omega$.

Next, the critical fault resistance $R_{f, c r}$ is defined by

$$
R_{f, \mathrm{cr}}=\frac{1}{3 \omega \sqrt{p C C_{n}}}
$$

When $R_{f}>R_{f, \mathrm{cr}}$, the derivative of $\left|\dot{I}_{0 n}\right|$ with respect to $p$ is negative, and thus $\left|\dot{I}_{0 n}\right|$ increases as $p$ decreases in the over-compensation region. This condition corresponds to the red curve in Fig. 2(f). On the other hand, if $R_{f}<R_{f, \mathrm{cr}},\left|\dot{I}_{0 n}\right|$ will decrease as $p$ decreases, which corresponds to the red curves in Figs. 2(a)-(e).

According to above analysis, both the ZSC magnitude $\left|\dot{I}_{0 i}\right|$ of sound feeder and the ZSC magnitude $\left|\dot{I}_{0 n}\right|$ of the faulted feeder would vary during the off-tuning adjustment of the ASC with significantly different adjustment trajectories: in general, $\left|\dot{I}_{0 i}\right|$ of the sound feeder is a symmetric about $p=0$, while $\left|\dot{I}_{0 n}\right|$ of the faulted feeder has more complex and asymmetrical characteristics.

\section{Proposed Faulted FeEder Identification Method}

\section{A. Principle of Faulted Feeder Identification}

Once an SPG fault occurs, the ASC will be tuned to compensate for the capacitive earth-fault current rapidly and the transient fault arc may be self-extinguished. After a short period of time, typically tens of fundamental cycles, the ASC would be adjusted to reduce the self-produced inductive current.
Meanwhile, the neutral-to-ground voltage is monitored to determine if the SPG fault has been cleared. If the fault has been cleared, the distribution network will automatically return to the normal operating mode. Otherwise, the ZSC variations will be used to identify the faulted feeder during this active off-tuning adjustment of the ASC.

Based on the ATM of the steady-state ZSCs presented in Section II, a similarity measure of the adjustment trajectories of ZSCs of all feeders shall be executed and the adjustment trajectory similarity will be used to identify the faulted feeder. The adjustment trajectories of two sound lines have a high degree of similarity, while the adjustment trajectories similarity between the currents of a sound line and a faulted line is low. Therefore, the similarity measurement of two adjustment trajectories of any two feeders in the ATM can be employed to establish the fault identification criterion.

\section{B. Similarity Measure for the Adjustment Trajectory Matrix}

Conventional similarity measuring methods to obtain the shape similarity of the captured ZSC variation signals are based on the cosine similarity [20] and HD similarity [21]. For these methods, a massive volume of sampling data needs to be collected and used as the input parameters. However, increasing the sampling data of the ZSCs with different compensation degrees would significantly increase the computational burden of the adjustment strategy of the ASC. In addition, it would cause potential hazards to both the ASC and the distribution lines. Therefore, it is preferable to use a small set of data to carry out the similarity measure for the problem at hand. In [22-24], GRA has demonstrated its ability to obtain the 
similarity measurement with high precision between two selected sequences with limited and incomplete information. Hence, GRA is well-suitable for measuring the similarity between two sampled ZSC variation signals of any two feeders, and the method is described below.

The power-frequency component magnitude of the ZSC of the $i$-th feeder under the compensation of $p_{m}$ is represented by $x_{\mathrm{i}}^{*}(m)$, where $m \in\{1,2, \cdots, n\}$ is the index of the compensation stage. Hence, in the proposed ASC adjustment scheme with $n$ compensation stages, the compensation degree $p$ would be controlled to $p_{1}, p_{2}, \ldots, p_{n}$. Hence, the corresponding adjustment trajectory, $x_{i}^{*}$, of the ZSC of the $i$-th feeder is expressed as,

$$
x_{i}^{*}=\left[\begin{array}{llll}
x_{i}^{*}(1) & x_{i}^{*}(2) & \cdots & x_{i}^{*}(n)
\end{array}\right]
$$

If $l$ feeders are connected to the same bus, the corresponding ATM of the ZSCs of all the feeders is given by

$$
X^{*}=\left[\begin{array}{cccc}
x_{1}^{*}(1) & x_{1}^{*}(2) & \cdots & x_{1}^{*}(n) \\
\vdots & \vdots & \vdots & \vdots \\
x_{i}^{*}(1) & x_{i}^{*}(2) & \cdots & x_{i}^{*}(n) \\
\vdots & \vdots & \vdots & \vdots \\
x_{l}^{*}(1) & x_{l}^{*}(2) & \cdots & x_{l}^{*}(n)
\end{array}\right]
$$

Three of the most commonly used GRA techniques, including Deng's GRA, the absolute GRA, and the slope GRA, are adopted as the methods for similarity measurement. The algorithms are described and compared in Table I.

A comparative study of the three typical GRA algorithms is carried out. Consider an SPG fault occurred at Feeder 4 of a substation with six feeders under different fault resistances and different compensation degrees. The calculated similarity measurements by applying the three GRA algorithms are shown in Table II.

TABLE I

\begin{tabular}{|c|c|c|c|}
\hline Method & Equations & Parameters Definitions & Description \\
\hline $\begin{array}{l}\text { Deng's } \\
\text { GRA }\end{array}$ & $\begin{array}{c}r_{i-j}^{\text {classical }}=\frac{1}{n} \sum_{k=1}^{n} \xi_{i-j}(k) \\
\xi_{i-j}(k)=\frac{\Delta \min +\rho \Delta \max }{\Delta_{i-j}(k)+\rho \Delta \max }\end{array}$ & $\begin{array}{c}\Delta \min =\min _{i} \min _{k}\left|x_{i}^{\mathrm{eq}}(k)-x_{j}^{\mathrm{eq}}(k)\right| \\
\Delta \max =\max _{i} \max _{k}\left|x_{i}^{\mathrm{eq}}(k)-x_{j}^{\mathrm{eq}}(k)\right| \\
\Delta_{i-j}(k)=\left|x_{i}^{\mathrm{eq}}(k)-x_{j}^{\mathrm{eq}}(k)\right| \\
x_{i}^{\mathrm{eq}}(k)=l \cdot x_{i}^{*}(k) / \sum_{i=1}^{l} x_{i}^{*}(k)\end{array}$ & $\begin{array}{l}\text { High computational efficiency, good } \\
\text { adaptivity for non-oscillatory signal } \\
\text { similarity measurement }\end{array}$ \\
\hline $\begin{array}{c}\text { Absolute } \\
\text { GRA }\end{array}$ & $r_{i-j}^{\text {absolute }}=\frac{1}{n-1} \sum_{k=2}^{n} \frac{1}{1+\left|\Delta y_{i}(k)-\Delta y_{j}(k)\right|}$ & $\begin{array}{c}\Delta y_{i}(k)=y_{i}(k+1)-y_{i}(k) \\
\Delta y_{j}(k)=y_{j}(k+1)-y_{j}(k) \\
y_{i}(k)=x_{i}^{*}(k) / x_{i}^{*}(1) \\
y_{j}(k)=x_{j}^{*}(k) / x_{j}^{*}(1)\end{array}$ & $\begin{array}{c}\text { Gradient based similarity } \\
\text { measurement, suitable for similarity } \\
\text { measurement of vibration signal }\end{array}$ \\
\hline $\begin{array}{l}\text { Slope } \\
\text { GRA }\end{array}$ & $\begin{array}{c}r_{i-j}^{\text {slope }}=\frac{1}{n-1} \sum_{k=1}^{n-1} r_{i-j}^{\text {slope }}(k) \\
r_{i-j}^{\text {slope }}(k)=\frac{\operatorname{sgn}\left(\Delta x_{i}^{*}(k), \Delta x_{j}^{*}(k)\right) \times\left[1+\left|\frac{\Delta x_{i}^{*}(k)}{\bar{x}_{i}^{*}}\right|\right.}{1+\left|\frac{\Delta x_{i}^{*}(k)}{\bar{x}_{i}^{*}}\right|+\left|\frac{\Delta x_{i}^{*}(k)}{\bar{x}_{i}^{*}}-\frac{\Delta x_{j}^{*}(k)}{\bar{x}_{j}^{*}}\right|}\end{array}$ & $\begin{array}{c}\operatorname{sgn}\left(\Delta x_{i}^{*}(k), \Delta x_{j}^{*}(k)\right)= \begin{cases}1 & \Delta x_{i}^{*}(k) \times \Delta x_{j}^{*}(k) \geq 0 \\
-1 & \Delta x_{i}^{*}(k) \times \Delta x_{j}^{*}(k)<0\end{cases} \\
\left\{\begin{array}{l}\Delta x_{i}^{*}(k)=x_{i}^{*}(k+1)-x_{i}^{*}(k) \\
\Delta x_{j}^{*}(k)=x_{j}^{*}(k+1)-x_{j}^{*}(k)\end{array}\right. \\
\bar{x}_{i}^{*}=\frac{1}{n} \sum_{k=1}^{n} x_{i}^{*}(k), \bar{x}_{j}^{*}=\frac{1}{n} \sum_{k=1}^{n} x_{j}^{*}(k)\end{array}$ & $\begin{array}{l}\text { Polarity-dependent similarity } \\
\text { measurement, suitable for similarity } \\
\text { measurement of rapid fluctuation } \\
\text { signals with positive and negative } \\
\text { samples }\end{array}$ \\
\hline
\end{tabular}

COMPARISON OF THREE TYPICAL GRA ALGORITHMS

Note: Similarity measurement between the $i$-th and the $j$-th adjustment trajectories, represented by $x_{i}{ }^{*}$ and $x_{j}{ }^{*}$

TABLE II

SimiLARITY MEASUREMENT RESULTS UNDER DiFFERENT GRA IMPLEMENTATION ALGORITHMS

\begin{tabular}{c|c|c|c|c|c}
\hline \hline $\begin{array}{c}\text { Similarity } \\
\text { Algorithm }\end{array}$ & Fault Condition & $\begin{array}{c}\text { Fault } \\
\text { Resistance }\end{array}$ & $\begin{array}{c}\text { Adjustment Time of } \\
\text { ASC }\end{array}$ & $\begin{array}{c}\text { Similarity Measurement Result } \\
r_{1}\left(r_{1-2}, r_{1-3}, r_{1-4}, r_{1-5}, r_{1-6}\right)\end{array}$ & Performance \\
\hline \multirow{3}{*}{ Deng' GRA } & Condition 1 & High & 4 & {$[0.9988,0.9995, \underline{0.5372,0.9986,0.9989]}$} & Good \\
& Condition 1 & High & 2 & {$[0.9982,0.9997, \underline{0.5122}, 0.9971,0.9984]$} \\
& Condition 2 & Low & 4 & {$[0.9967,0.9976, \underline{0.5817}, 0.9951,0.9951]$} & Good \\
Absolute & Condition 1 & High & 4 & {$[0.9998,0.9999, \underline{0.9364}, 0.9998,0.9998]$} & Good \\
GRA & Condition 1 & High & 2 & {$[0.9998,1.0000, \underline{0.9257}, 0.9998,0.9999]$} & Weak \\
& Condition 2 & Low & 4 & {$[0.9996,0.9997, \underline{0.9278}, 0.9995,0.9994]$} & Weak \\
Slope GRA & Condition 1 & High & 4 & {$[0.9998,0.9999, \underline{0.4694,0.9998,0.9998]}$} & Weak \\
& Condition 1 & High & 2 & {$[0.9998,1.0000,-\underline{0.0126}, 0.9998,0.9999]$} & Good \\
\hline \hline
\end{tabular}

Note: 1) Fault Condition 1 represents an SPG fault occurred on Feeder 4 at a distance of $25 \mathrm{~km}$ from the bus and $R_{f}=1000 \Omega$; 2) Fault Condition 2 represents an SPG fault occurred on Feeder 4 at a distance of $5 \mathrm{~km}$ from the bus and $R_{f}=1 \Omega ; 3$ ) The symbol $r_{i-j}$ represents the similarity of the adjustment trajectories of the ZSCs between the $i$-th and the $j$-th feeders. 
Recall that the similarity is defined within $[-1,1]$. A score of 1 indicates that the analyzed two adjustment trajectories are identical, where a score of -1 represents that one adjustment trajectory is a reverse curve of the other. As shown in Table II, Deng's GRA algorithm with low computational cost demonstrates a good performance. The similarity results of the Deng's GRA algorithm are very robust when the fault resistance or the number of compensation degrees varies in a wide range. Therefore, Deng's GRA algorithm is adopted for the study in the rest of the work.

By using Deng's GRA algorithm, the similarity of the adjustment trajectories of the ZSC between the $i$-th and the $j$-th feeders can be calculated as,

$$
r_{i-j}=\frac{1}{n} \sum_{k=1}^{n} \xi_{i-j}(k)
$$

where the grey relational degree coefficient $\xi_{i-j}(k)$ is defined by

$$
\xi_{i-j}(k)=\frac{\Delta \min +\rho \Delta \max }{\Delta_{i-j}(k)+\rho \Delta \max }
$$

with

$$
\begin{gathered}
\Delta \min =\min _{i} \min _{k}\left|x_{i}^{\mathrm{eq}}(k)-x_{j}^{\mathrm{eq}}(k)\right| \\
\Delta \max =\max _{i} \max _{k}\left|x_{i}^{\mathrm{eq}}(k)-x_{j}^{\mathrm{eq}}(k)\right| \\
\Delta_{i-j}(k)=\left|x_{i}^{\mathrm{eq}}(k)-x_{j}^{\mathrm{eq}}(k)\right|
\end{gathered}
$$

In (8), $\rho$ is the resolution coefficient. In (9)-(11), $x_{i}^{\mathrm{eq}}(k)$ represents the normalized value of the $k$-th element of the $i$-th adjustment trajectory. Because the maximum value may be equal to the minimum value in some adjustment trajectory when an SPG fault occurs with zero fault resistance, an equalization algorithm is used to normalize the current sequence instead of the relativization algorithm as,

$$
x_{i}^{\mathrm{eq}}(k)=\frac{x_{i}^{*}(k)}{\frac{1}{l} \sum_{i=1}^{l} x_{i}^{*}(k)}
$$

\section{Faulted Feeder Identification Criterion}

The similarity of the adjustment trajectories between the $i$-th feeder and other feeders can be obtained using (8), i.e.,

$$
r_{i}=\left[\begin{array}{llll}
r_{i-1} & r_{i-2} & \ldots & r_{i-l}
\end{array}\right]
$$

The average similarity related to the adjustment trajectory of $i$-th feeder is defined by,

$$
\overline{r_{i}}=\frac{1}{l-1} \sum_{j=1, j \neq i}^{l} r_{i-j}
$$

As mentioned earlier, the adjustment trajectories of the two sound lines have a higher similarity degree, while the adjustment trajectories similarity between one sound line and a faulted line is much lower. ZSC signal similarity between one sound line and a faulted line is represented by $r_{f}$, while the ZSC signal similarity between two sound lines is about 1.0. Hence the obtained average similarity of the faulted feeder is shown as,

$$
\bar{r}_{n}=\sum_{j=1, j \neq n}^{l} r_{n-j} /(l-1)=r_{f}
$$

The obtained average similarity of the sound feeder is

$$
\overline{r_{i}}=\sum_{j=1, j \neq i}^{l} r_{i-j} /(l-1)=1-\left(1-r_{f}\right) /(l-1)
$$

The relationship between $r_{n}$ and $r_{i}$ is,

$$
\bar{r}_{n}=r_{f}=\left[(l-2) r_{f}+r_{f}\right] /(l-1) \ll\left[l-2+r_{f}\right] /(l-1)=\bar{r}_{i}
$$

Hence, the obtained average similarity of the faulted feeder is much lower than that of any sound feeder.

The final faulted feeder identification criterion is constructed by the determination coefficient $\lambda_{i}$ and the threshold $\lambda_{\text {set }}$, i.e.

$$
\begin{gathered}
\lambda_{i}=\sum_{j=1, j \neq i}^{l}\left|\bar{r}_{i}-\bar{r}_{j}\right| \\
\lambda_{\text {set }}=K_{\mathrm{re}} \sqrt{\frac{1}{l-1}\left(\sum_{i=1}^{l} \lambda_{i}-\max \left(\lambda_{i}\right)\right) \max \left(\lambda_{i}\right)}
\end{gathered}
$$

where the setting coefficient $K_{\mathrm{re}}$ is 1.4 in this paper.

The flow chart of the proposed faulted feeder identification method in RGDNs is shown in Fig. 3. To summarize, when an SPG fault occurs in the RGDN, the zero-sequence bus voltage is used to initialize the faulted feeder identification process. The power-frequency magnitude sequences of the ZSC of any feeder are recorded to construct the ATM under $n$ different compensation degrees. Next, the similarity of any two adjustment trajectories in the ATM is measured by Deng's GRA. The final faulted feeder identification criterion is established by the determination coefficient $\lambda_{i}$ and the identification threshold $\lambda_{\text {set. }}$.

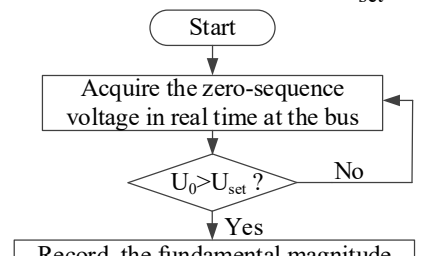

Record the fundamental magnitude sequences of zero-sequence currents of all feeders to construct the adjustment trajectory matrix under $n$ number of different compensation degrees

Measure the similarity of any two adjustment trajectories by using Deng's GRA

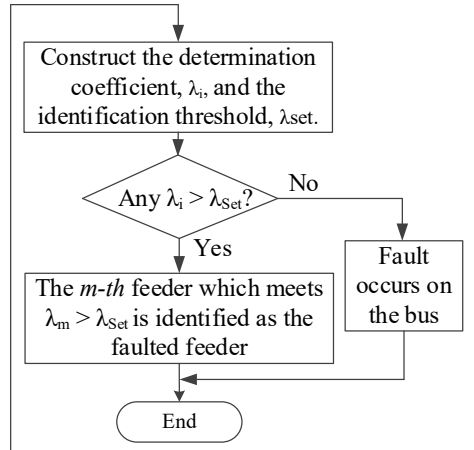

Fig. 3. Flow chart of the proposed faulted feeder identification method in RGDNs.

The sensitivity $K_{\text {sen }}$ of the faulted identification method is used to represent the confidence coefficient of the selected result and it is defined as $K_{\text {sen }}=\lambda_{n} / \lambda_{\text {set. Here }} \lambda_{n}$ is the determination coefficient of the selected faulted feeder $n$. Random noise, measuring error, and truncation error in the digital identification device may cause a decrease in the determination coefficient $\lambda_{n}$ of the faulted Feeder $n$ and an increase in identification threshold $\lambda_{\text {set. }}$. Therefore, the smaller the sensitivity $K_{\text {sen }}$ is, the higher the probability of incorrect identification is. The larger the sensitivity $K_{\text {sen }}$ is, the better the adaptive capacity of the proposed method is under different fault conditions or with different feeder parameters. 


\section{CASe Study}

\section{A. System Configuration}

The schematic of a typical $10-\mathrm{kV}$ distribution system used for case study is shown in Fig. 4. The system contains two overhead lines, three underground cables, and one mixed feeder consisting of an overhead line and an underground cable. The model was implemented in PSCAD/EMTDC environment. The parameters of the overhead lines and underground cables were obtained from [25].

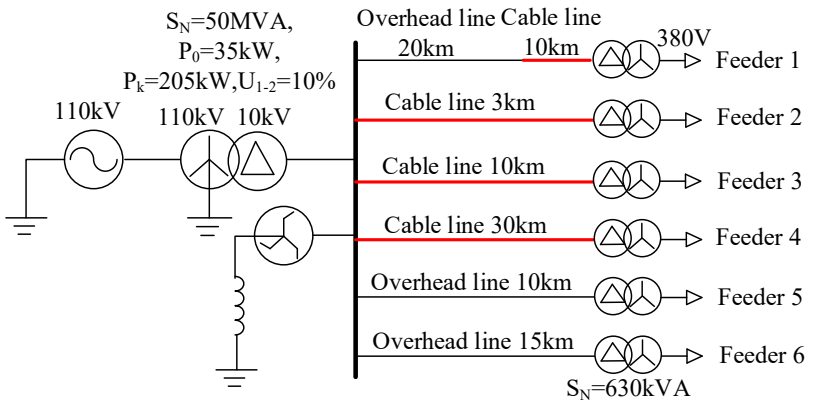

Fig. 4. Schematic diagram of a typical resonant grounding distribution network used in case study.

According to the parameters of the distribution system, the shunt capacitance of Feeder 5 is the lowest amongst all feeders, and it accounts for $0.67 \%$ of the total capacitance. While the shunt capacitance of Feeder 4 is the highest, accounting for $54.89 \%$ of the total capacitance.

\section{B. Simulation Results and Discussion}

The ZSCs of the feeders were extracted and analyzed with different compensation degrees. In this case, the SPG faults were set to occur at different locations of Feeder 1 to Feeder 6 , with different fault transition resistances $R_{f}$.

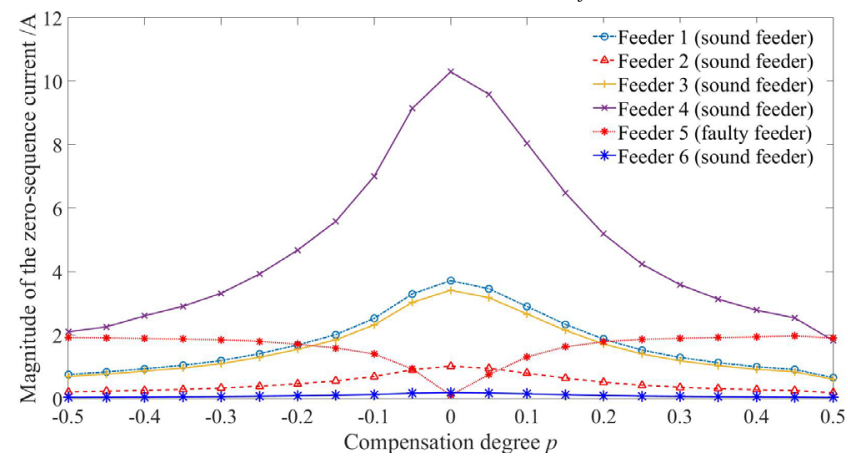

Fig. 5. The adjustment trajectories of the ZSCs of all feeders when compensation degree changes (fault occurs on Feeder 5, $R_{f}=1000 \Omega$ ).

When an SPG fault occurs at the end of Feeder 5 with $R_{f}=$ $1000 \Omega$, the adjustment trajectories of different feeders are obtained and shown in Fig. 5 with $p$ varying from -0.5 to 0.5 . It shows that both the magnitudes of ZSCs of the faulted and the sound lines vary significantly as $p$ varies. As $p$ decreases from 0.5 to -0.5 , the magnitude of the ZSC of the sound line increases first, while it starts to decrease at $p=0$. This trend is opposite to that of the ZSC of the faulted line. If the faulted feeder identification method proposed in [15] is used, Feeder 4 would be incorrectly determined as the faulted line. This is due to that the magnitude variation of the ZSC of Feeder 4 is the most significant when the tap position is adjusted to the next one.

The residual fault current is directly proportional to the compensation degree. Once an SPG fault occurs, the ASC would be adjusted rapidly to keep the compensation degree in zero state. To avoid arc reignition, the compensation degree should not be adjusted far away from zero. Considering the measurement error of line-to-capacitance in the field, we give a high priority in the range of $[-0.2,0.2]$ of compensation degrees in the test. Here, $p$ is controlled to vary between $\pm 10 \%$ during the ASC adjustment process. The ATM of all feeders is monitored and obtained at $p= \pm 10 \%, \pm 5 \%$, and 0 , respectively.

The faulted feeder identification results by using Deng's GRA are shown in Table III. $L_{m}$ represents the serial number of the faulted feeder and $X_{f}$ is the distance from fault point to the substation bus. The coefficients $\lambda_{1}-\lambda_{6}$ of the six feeders are obtained by using (15), and the identification threshold, $\lambda_{\text {set, }}$ is calculated by (16). In this example, the SPG fault occurs on Feeder 1 with $R_{f}=1000 \Omega$. The fault is located $25 \mathrm{~km}$ away from the substation bus, and the determination coefficients $\lambda_{1}-\lambda_{6}$ are $1.61404,0.32292,0.323,0.32292,0.323$, and 0.32292 , respectively. The corresponding calculated threshold is $\lambda_{\text {set }}=$ 1.011. As $\lambda_{1}>\lambda_{\text {set }}$ and all other determination coefficients are less than $\lambda_{\text {set }}$, Feeder 1 is identified as the faulted feeder. In this case, the sensitivity for the identification is $\lambda_{1} / \lambda_{\text {set }}=1.60$. Other SPG faults are located at different positions on each feeder with different fault resistances. The corresponding identification results are also given in Table III and it shows the faulted feeder has been reliably identified regardless of the fault conditions.

Nowadays, modern single-phase inverter-based ASCs [26], magnetically controlled reactor-based flexible ASCs [27], and other types of ASCs are proposed and applied in the field. The recorded ZSCs data in the field during the active adjustment of the flexible ASC is next used to verify our proposed method. The ZSC waveforms are depicted in Fig. 6. The inductance of the ASC is first adjusted at the time $t=0.041 \mathrm{~s}$ and the duration is $10 \mathrm{~ms}$. The second adjustment of the ASC starts from $t=0.1$ $\mathrm{s}$ and it ends at $t=0.108 \mathrm{~s}$. The current flowing through the ASC as well as the ZSCs of the faulted and sound feeders are recorded by the ASC. Distortion can be readily observed in the recorded ZSC of the faulted feeder because the ASC device generates harmonic components. By using Fourier analysis to extract the fundamental frequency component, it is found that the obtained determination coefficients $\lambda_{1}-\lambda_{4}$ are 0.3317 , $0.1539,0.1320$, and 0.1320 , respectively. As the corresponding threshold is 0.300 , Feeder 1 is correctly identified as the faulted feeder based on the measured data in the field.

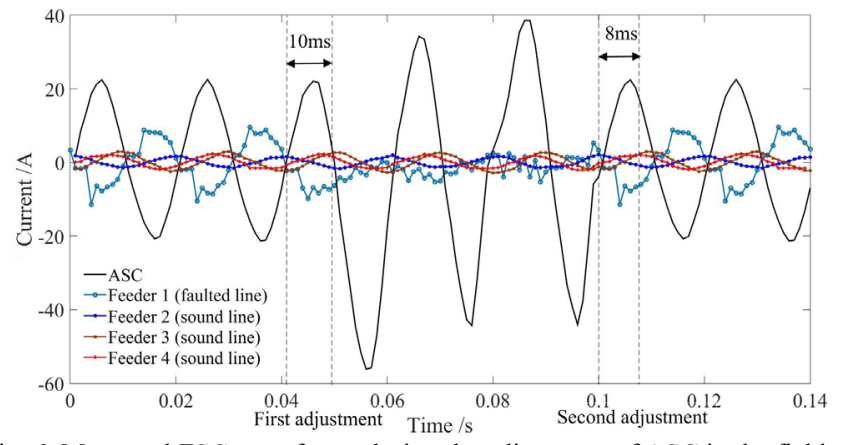

Fig. 6. Measured ZSC waveforms during the adjustment of ASC in the field. 
TABLE III

FAULTED FEEDER IDENTIFICATION RESULTS

\begin{tabular}{|c|c|c|c|c|c|c|}
\hline$L_{m}$ & $X_{f}(\mathrm{~km})$ & $R_{f}(\Omega)$ & $\lambda_{i}\left(\left[\lambda_{1}, \lambda_{2}, \lambda_{3}, \lambda_{4}, \lambda_{5}, \lambda_{6}\right]\right)$ & $\lambda_{\text {set }}$ & Result & Sensitivity \\
\hline \multirow{10}{*}{1} & 0.1 & 1 & {$[1.58170,0.31634,0.31634,0.31634,0.31634,0.31634]$} & 0.990 & Right & 1.60 \\
\hline & 0.1 & 50 & {$[1.60508,0.32124,0.3214,0.32132,0.3212,0.3212]$} & 1.005 & Right & 1.60 \\
\hline & 0.1 & 1000 & {$[1.61188,0.32268,0.32276,0.3226,0.32268,0.3226]$} & 1.009 & Right & 1.60 \\
\hline & 5 & 1 & {$[1.58406,0.33366,0.32414,0.32414,0.32454,0.32478]$} & 1.006 & Right & 1.57 \\
\hline & 15 & 50 & {$[1.61396,0.3238,0.3238,0.32364,0.32364,0.32364]$} & 1.012 & Right & 1.59 \\
\hline & 20 & 200 & {$[1.60474,0.33042,0.3257,0.3249,0.3249,0.32538]$} & 1.013 & Right & 1.58 \\
\hline & 20 & 500 & {$[1.61588,0.32468,0.32476,0.32476,0.32468,0.32676]$} & 1.015 & Right & 1.59 \\
\hline & 25 & 1000 & {$[1.61404,0.32292,0.323,0.32292,0.323,0.32292]$} & 1.011 & Right & 1.60 \\
\hline & 25 & 3000 & {$[1.359,0.27324,0.27324,0.27276,0.27348,0.27276]$} & 0.853 & Right & 1.59 \\
\hline & 25 & 5000 & {$[\underline{1.40514}, 0.28186,0.28174,0.28194,0.28266,0.28174]$} & 0.881 & Right & 1.59 \\
\hline \multirow{7}{*}{2} & 1 & 1 & {$[0.3417,1.70286,0.3419,0.34138,0.34126,0.34126]$} & 1.068 & Right & 1.59 \\
\hline & 1 & 50 & {$[0.34248, \underline{1.71128}, 0.34244,0.3424,0.3424,0.34252]$} & 1.072 & Right & 1.60 \\
\hline & 1 & 200 & {$[0.34402, \underline{1.71806}, 0.34386,0.34378,0.3439,0.34378]$} & 1.076 & Right & 1.60 \\
\hline & 1 & 500 & {$[0.35926, \underline{1.79414}, 0.35918,0.35906,0.35906,0.3591]$} & 1.124 & Right & 1.60 \\
\hline & 1 & 1000 & {$[0.33992,1.69788,0.33996,0.33992,0.33996,0.33996]$} & 1.064 & Right & 1.60 \\
\hline & 1 & 3000 & {$[0.40792,2.0376,0.40836,0.40808,0.40792,0.40796]$} & 1.276 & Right & 1.60 \\
\hline & 1 & 5000 & {$[0.31588,1.57584,0.31588,0.31588,0.31696,0.31596]$} & 0.988 & Right & 1.59 \\
\hline \multirow{7}{*}{3} & 3 & 1 & {$[0.31686,0.31666,1.58238,0.31678,0.3167,0.31666]$} & 0.991 & Right & 1.60 \\
\hline & 5 & 50 & {$[0.32044,0.3204,1.6002,0.3204,0.32028,0.32028]$} & 1.002 & Right & 1.60 \\
\hline & 5 & 200 & {$[0.3217,0.32162, \underline{1.60646}, 0.32174,0.32158,0.32158]$} & 1.006 & Right & 1.60 \\
\hline & 5 & 500 & {$[0.3236,0.3236,1.6168,0.32364,0.3236,0.32372]$} & 1.013 & Right & 1.60 \\
\hline & 7 & 1000 & {$[0.31582,0.31586,1.57642,0.31578,0.31598,0.31578]$} & 0.988 & Right & 1.60 \\
\hline & 10 & 3000 & {$[0.28044,0.28048, \underline{1.39788}, 0.28056,0.28108,0.28044]$} & 0.877 & Right & 1.59 \\
\hline & 10 & 5000 & {$[0.27174,0.27166,1.34738,0.27506,0.27166,0.27214]$} & 0.848 & Right & 1.59 \\
\hline \multirow{7}{*}{4} & 5 & 1 & {$[0.33256,0.33112, \overline{0.33064}, \underline{1.64192}, 0.33024,0.33024]$} & 1.032 & Right & 1.59 \\
\hline & 15 & 50 & {$[0.33296,0.33304,0.33292, \underline{1.66368}, 0.33292,0.33312]$} & 1.042 & Right & 1.60 \\
\hline & 15 & 200 & {$[0.3375,0.3375,0.34582,1.6575,0.33774,0.33774]$} & 1.050 & Right & 1.58 \\
\hline & 15 & 500 & {$[0.323,0.32228,0.33588,1.56556,0.32236,0.32228]$} & 0.999 & Right & 1.57 \\
\hline & 25 & 1000 & {$[0.36382,0.36362,0.36362, \underline{1.81522}, 0.3641,0.36398]$} & 1.138 & Right & 1.60 \\
\hline & 25 & 3000 & {$[0.28136,0.28224,0.28184, \underline{1.40328}, 0.28152,0.28136]$} & 0.880 & Right & 1.59 \\
\hline & 25 & 5000 & {$[0.38268,0.3842,0.38484,1.79736,0.41432,0.38268]$} & 1.172 & Right & 1.53 \\
\hline \multirow{7}{*}{5} & 3 & 1 & {$[0.29384,0.29384,0.29424,0.294,1.46628,0.29388]$} & 0.919 & Right & 1.60 \\
\hline & 5 & 50 & {$[0.29424,0.29428,0.29428,0.29444, \underline{1.47004}, 0.29424]$} & 0.921 & Right & 1.60 \\
\hline & 5 & 200 & {$[0.30128,0.2962,0.29612,0.29604, \underline{1.4636}, 0.29604]$} & 0.923 & Right & 1.58 \\
\hline & 5 & 500 & {$[0.2884,0.28808,0.28964,0.28808,1.43468,0.28808]$} & 0.901 & Right & 1.59 \\
\hline & 7 & 1000 & {$[0.31942,0.3197,0.31946,0.31942, \underline{1.59562}, 0.31946]$} & 1.000 & Right & 1.60 \\
\hline & 10 & 3000 & {$[0.30806,0.30794,0.30802,0.30794,1.53854,0.30794]$} & 0.964 & Right & 1.60 \\
\hline & 10 & 5000 & {$[0.2905,0.29726,0.2905,0.2907, \underline{1.4307,0.29054]}$} & 0.905 & Right & 1.58 \\
\hline \multirow{7}{*}{6} & 3 & 1 & {$[0.32286,0.32294,0.32294,0.32294,0.32286,1.61086]$} & 1.010 & Right & 1.60 \\
\hline & 5 & 50 & {$[0.27728,0.27724,0.27724,0.27752,0.27764,1.38388]$} & 0.867 & Right & 1.60 \\
\hline & 5 & 200 & {$[0.27538,0.27522,0.27522,0.27514,0.27514,1.37426]$} & 0.861 & Right & 1.60 \\
\hline & 5 & 500 & {$[0.28174,0.28174,0.28174,0.28186,0.28182,1.40754]$} & 0.882 & Right & 1.60 \\
\hline & 7 & 1000 & {$[0.31808,0.31848,0.31808,0.31804,0.31804,1.58848]$} & 0.995 & Right & 1.60 \\
\hline & 15 & 3000 & {$[0.31282,0.31298,0.3129,0.31282,0.3129, \underline{1.5633}]$} & 0.979 & Right & 1.60 \\
\hline & 15 & 5000 & {$[0.3043,0.30434,0.3045,0.30522,0.3043,1.51746]$} & 0.952 & Right & 1.59 \\
\hline \multirow{7}{*}{ Bus } & & 1 & {$[0.36546,0.3733,0.28026,0.33062,0.33062,0.28026]$} & 0.482 & Right & \\
\hline & & 50 & {$[0.26352,0.3424,0.3428,0.24572,0.24572,0.25176]$} & 0.426 & Right & \\
\hline & & 200 & {$[0.27482,0.22834,0.34562,0.22834,0.33154,0.31546]$} & 0.432 & Right & \\
\hline & - & 500 & {$[0.13872,0.1896,0.10932,0.14772,0.10932,0.13572]$} & 0.218 & Right & - \\
\hline & & 1000 & {$[0.27624,0.17544,0.29448,0.15324,0.17056,0.15324]$} & 0.327 & Right & \\
\hline & & 3000 & {$[0.23474,0.11722,0.12926,0.11722,0.1951,0.1605]$} & 0.257 & Right & \\
\hline & & 5000 & {$[0.11786,0.32202,0.11182,0.11182,0.1277,0.17802]$} & 0.286 & Right & \\
\hline
\end{tabular}

TABLE IV

FAULTED FEEDER IDENTIFICATION RESULTS UNDER DifFERENT ADJUSTMENT SCHEMES OF THE ASC

\begin{tabular}{|c|c|c|c|c|c|}
\hline Fault Conditions & Scheme of Peterson Coil Adjustment & $\lambda_{i}\left(\left[\lambda_{1}, \lambda_{2}, \lambda_{3}, \lambda_{4}, \lambda_{5}, \lambda_{6}\right]\right)$ & $\lambda_{\text {set }}$ & Result & Sensitivity \\
\hline $\begin{array}{c}\text { Feeder } 4, \\
X_{f}=25 \mathrm{~km}, \\
R_{f}=1000 \Omega\end{array}$ & $\begin{array}{c}{[+20 \%,+15 \%,+10 \%,+5 \%, 0]} \\
{[+15 \%,+10 \%,+5 \%, 0,-5 \%]} \\
{[+10 \%, 5 \%, 0,-5 \%,-10 \%]} \\
{[+5 \%, 0,-5 \%,-10 \%,-15 \%]} \\
{[0,-5 \%,-10 \%,-15 \%,-20 \%]}\end{array}$ & $\begin{array}{c}{[0.34736,0.34712,0.34712, \underline{1.7338}, 0.34712,0.34724]} \\
{[0.3608,0.3606,0.3606, \underline{1.8008}, 0.36088,0.36088]} \\
{[0.36382,0.36362,0.36362, \underline{1.81522}, 0.3641,0.36398]} \\
{[0.3303,0.3303,0.3303, \underline{1.65042}, 0.33042,0.3303]} \\
{[0.33188,0.33188,0.33204,1.65864,0.33208,0.33188]}\end{array}$ & $\begin{array}{l}1.086 \\
1.128 \\
1.138 \\
1.034 \\
1.039\end{array}$ & $\begin{array}{l}\text { Right } \\
\text { Right } \\
\text { Right } \\
\text { Right } \\
\text { Right }\end{array}$ & $\begin{array}{l}1.60 \\
1.60 \\
1.60 \\
1.60 \\
1.60\end{array}$ \\
\hline
\end{tabular}


TABLE V

FAULTED FEEDER IDENTIFICATION RESULTS WITH ADDED GAUSSIAN-WHITE NOISES

\begin{tabular}{|c|c|c|c|c|c|}
\hline Fault Conditions & Gaussian Noise (dB) & $\lambda_{i}\left(\left[\lambda_{1}, \lambda_{2}, \lambda_{3}, \lambda_{4}, \lambda_{5}, \lambda_{6}\right]\right)$ & $\lambda_{\text {set }}$ & Result & Sensitivity \\
\hline & No noise added & {$[0.31582,0.31586,1.57642,0.31578,0.31598,0.31578]$} & 0.988 & Right & 1.60 \\
\hline & 50 & {$[0.31358,0.31358, \underline{1.50166}, 0.31482,0.32766,0.3173]$} & 0.966 & Right & 1.55 \\
\hline Feeder 3, & 45 & {$[0.3092,0.3092, \underline{1.51508}, 0.31552,0.3094,0.3116]$} & 0.961 & Right & 1.58 \\
\hline$X_{f}=7 \mathrm{~km}$, & 40 & {$[0.31012,0.32228, \overline{1.45304}, 0.31608,0.33052,0.31012]$} & 0.951 & Right & 1.53 \\
\hline \multirow[t]{3}{*}{$R_{f}=1000 \Omega$} & 35 & {$[0.29178,0.30326, \underline{1.3255}, 0.3093,0.31202,0.29178]$} & 0.885 & Right & 1.50 \\
\hline & 30 & {$[0.32634,0.28114,0.98602,0.32594,0.27882,0.27882]$} & 0.759 & Right & 1.30 \\
\hline & 25 & {$[0.27702,0.2791,1.0307,0.2711,0.24646,0.24646]$} & 0.730 & Right & 1.41 \\
\hline
\end{tabular}

TABLE VI

FAULT FEEDER IDENTIFICATION RESULTS UNDER DIFFERENT NUMBERS OF COMPENSATION DEGREES DURING THE ADJUSTMENT OF ASC

\begin{tabular}{|c|c|c|c|c|c|}
\hline Fault Conditions & $\begin{array}{l}\text { Number of the Compensation } \\
\text { Degrees During the Adjustment }\end{array}$ & $\lambda_{i}\left(\left[\lambda_{1}, \lambda_{2}, \lambda_{3}, \lambda_{4}, \lambda_{5}, \lambda_{6}\right]\right)$ & $\lambda_{\text {set }}$ & Result & Sensitivity \\
\hline \multirow{4}{*}{$\begin{array}{c}\text { Feeder } 4, \\
X_{f}=25 \mathrm{~km}, \\
R_{f}=1000 \Omega\end{array}$} & 3 & {$[0.38674,0.38674,0.38682,1.92672,0.38672,0.38754]$} & 1.2093 & Right & 1.59 \\
\hline & 5 & {$[0.36382,0.36362,0.36362, \underline{1.81522}, 0.3641,0.36398]$} & 1.1377 & Right & 1.59 \\
\hline & 7 & {$[0.35742,0.35726,0.35722,1.78482,0.35746,0.35722]$} & 1.1180 & Right & 1.60 \\
\hline & 9 & {$[0.35050,0.35050,0.35050,1.75142,0.35050,0.35054]$} & 1.097 & Right & 1.60 \\
\hline
\end{tabular}

\section{Sensitivity Analysis of the Proposed Faulted FEEDER IDENTIFICATION METHOD}

\section{A. Effect of Different Adjustment Scheme of the ASC}

In the previous section, all the feeders are assumed to be monitored at the specified compensation degrees. However, in practice this assumption may not be always valid and different compensation degrees may be adopted for the identification in the distribution network. To verify the effectiveness and robustness of the proposed method, simulation with different inductance adjustment schemes of the ASC at different sequences of compensation degrees were carried out. Suppose an SPG fault occurs on Feeder 4, the corresponding identification results are given in Table IV. It shows that in all conditions $\lambda_{4}$ of Feeder 4 is much larger than the determination coefficients of other feeders. Meanwhile, the sensitivity $\lambda_{4} / \lambda_{\text {set }}$ almost remains constant with different ASC adjustment schemes. Furthermore, the ratio of determination coefficient of sound feeder to the threshold is very low, which indicates the proposed method has high reliability. Therefore, the effectiveness of the proposed method is hardly affected by the adjustment schemes of the ASC.

\section{B. Effect of Random Noises}

The measured current waveforms are usually contaminated by random noises in the field. The noise tolerance is important for practical application of the fault feeder identification method, when the ZSC magnitude curves are used. Table V shows the determination coefficients all feeders when an SPG fault occurs on Feeder 3 when Gaussian-white noises are added. It can be observed that the determination coefficient $\lambda_{3}$ of Feeder 3 is always much higher than that of other feeders regardless of the level of the noise. The sensitivity of the faulted feeder identification, which is around 1.60, shows that Feeder 3 can be identified accurately despite the interference of the noise.

\section{Effect of the Number of Different Compensation Degrees}

As shown in (6), the proposed similarity measurement of the adjustment trajectories is conducted using $n$ different compensation degrees. The similarity can be obtained only when the number of the different compensation degrees is no less than three. Nevertheless, the ASC adjustment capability limits the total number of the different compensation degrees during the SPG fault.

Consider an SPG fault occurs on Feeder 4 with $R_{f}=1000 \Omega$ at the position of $25 \mathrm{~km}$ away from the substation bus. The determination coefficients are given in Table VI, along with the identification thresholds under different numbers of compensation degrees. The results show that the effectiveness of the proposed method and the obtained similarity are almost not affected by the number of compensation degrees.

\section{Effect of Different Similarity Measurement Algorithms}

To show the advantages of using GRA-based similarity analysis, comparison with the widely-used cosine similarity and HD similarity are given in this subsection. If an SPG fault occurs on Feeder 5, three-number different compensation degrees are used to test these similarity measurement algorithms, and the results for the Feeder 1 are given in Table VII.

For the cosine similarity method, the similarity of the current variations of one sound line and the faulted line is about 0.9974 . Since this value is very close to that of the two sound lines, it cannot be used to identify the faulted feeder. In this case, the HD algorithm may be effective because the obtained similarity of the current variations of a sound line and the faulted line is significantly different from that of the two sound lines. However, in practice, the HD algorithm is highly susceptible to noise as the similarity is represented by the maximum distance of a set to the nearest point in the other set.

TABLE VII

SIMILARITY ANALYSIS RESULTS USING DIFFERENT ALGORITHMS

\begin{tabular}{ccc} 
Fault Condition & Similarity Algorithm & $r_{1}\left(r_{1-2}, r_{1-3}, r_{1-4}, r_{1-5}, r_{1-6}\right)$ \\
\hline Feeder 4, & GRA & {$[0.9982,0.9997, \underline{0.5122}$,} \\
$X_{f}=25 \mathrm{~km}$, & Cosine similarity & $0.9971,0.9984]$ \\
$R_{f}=1000 \Omega$ & Hausdorff-distance & {$[0.0003,0.00005, \underline{0.1}$,} \\
& & $0.00028,0.00018]$ \\
\hline \hline
\end{tabular}


TABLE VIII

FAULTED FEEDER IDENTIFICATION RESULTS UNDER DiFFERENT NUMBERS OF FEEDERS

\begin{tabular}{|c|c|c|c|c|c|}
\hline Fault Condition & Number of feeders & $\lambda_{i}$ & $\lambda_{\text {set }}$ & Result & Sensitivity \\
\hline \multirow{4}{*}{$\begin{array}{c}\text { Feeder } 1, \\
X_{f}=25 \mathrm{~km}, \\
R_{f}=1000 \Omega\end{array}$} & 3 & {$[\underline{0.35435}, 0.1772,0.17725]$} & 0.351 & Right & 1.01 \\
\hline & 4 & {$[0.76707,0.25573,0.25573,0.25587]$} & 0.620 & Right & 1.24 \\
\hline & 5 & {$[1.1884,0.29722,0.29722,0.29722,0.29732]$} & 0.832 & Right & 1.43 \\
\hline & 6 & {$[1.61404,0.32292,0.323,0.32292,0.323,0.32292]$} & 1.011 & Right & 1.60 \\
\hline
\end{tabular}

TABLE IX

COMPARISON OF DIFFERENT FAULTED FEEDER IDENTIFICATION METHODS

\begin{tabular}{|c|c|c|c|}
\hline Method & Feature Matrix & $\begin{array}{l}\text { Faulted Feeder } \\
\text { Identification }\end{array}$ & Evaluation Result \\
\hline Proposed Method & $\begin{array}{c}\lambda_{i}\left(\left[\lambda_{1}, \lambda_{2}, \lambda_{3}, \lambda_{4}, \lambda_{5}, \lambda_{6}\right]\right), \lambda_{\text {set }} \\
{[0.25788,0.25732,1.27972,0.25708,0.25708,0.25892], 0.804}\end{array}$ & 3 & Right \\
\hline VCASC & $\begin{array}{c}\delta \mathrm{I}\left(\left[\left|\delta \mathrm{I}_{1}\right|,\left|\delta \mathrm{I}_{2}\right|,\left|\delta \mathrm{I}_{3}\right|,\left|\delta \mathrm{I}_{4}\right|,\left|\delta \mathrm{I}_{5}\right|,\left|\delta \mathrm{I}_{6}\right|\right]\right) \\
{[0.86,0.2376,0.671, \underline{2.363}, 0.02887,0.04333]}\end{array}$ & 4 & Wrong \\
\hline TGRA & $\begin{array}{c}\lambda_{i}\left(\left[\lambda_{1}, \lambda_{2}, \lambda_{3}, \lambda_{4}, \lambda_{5}, \lambda_{6}\right]\right), \lambda_{\text {set }} \\
{[-0.04722,0.10026,0.04854,-0.0309,0.05466,-0.12534],-0.5}\end{array}$ & bus & Wrong \\
\hline VCFH & $\begin{array}{c}\left.\left.\mathrm{I} 5\left(\left[\left|\mathrm{I} 5_{(1)}\right|,\left|\mathrm{I} 5_{(2)}\right|,\left|\mathrm{I} 5_{(3)}\right|, \mid \mathrm{I} 5_{(4)}\right),\left|\mathrm{I} 5_{(5)}\right|, \mid \mathrm{I} 5_{(6)}\right]\right]\right) \\
{[0.0136,0.0041,0.0122,0.0399,0.0005,0.0007]}\end{array}$ & 4 & Wrong \\
\hline WLPC & $\begin{array}{c}\operatorname{sgn}\left(I_{i}\right)\left(\left[\operatorname{sgn}\left(I_{1}\right), \operatorname{sgn}\left(I_{2}\right), \operatorname{sgn}\left(I_{3}\right), \operatorname{sgn}\left(I_{4}\right), \operatorname{sgn}\left(I_{5}\right), \operatorname{sgn}\left(I_{6}\right)\right]\right) \\
{[-,+,-,+,++]}\end{array}$ & / & Failed \\
\hline
\end{tabular}

Note: VCFH represents the faulted feeder identification method based on the comparison of fifth harmonic components of ZSCs, WLPC represents the method based on wavelet analysis and polarity comparison, VCASC represents the method based on variation comparison under ASC adjustment [15], and TGRA represents the method based on transient signals and grey relational analysis [22].

On the contrary, for the proposed GRA-based algorithm, the similarity of the ZSC variations of two sound lines is about 1.0, while the similarity of the current variations of a sound line and the faulted line is only 0.5122 . The results show that GRA algorithm can be readily used to identify the faulted feeder and it outperforms the other two algorithms with excellent noise tolerance.

\section{E. Effect of the Number of Feeders}

Next, the sensitivity $K_{\text {sen }}$ with different numbers of feeders is analyzed for the verification of the proposed method. The identification threshold $\lambda_{\text {set }}$ is calculated by

$$
\begin{aligned}
\lambda_{\text {set }} & =K_{\mathrm{re}} \cdot \sqrt{\frac{1}{l-1}\left(\sum_{i=1}^{l} \lambda_{i}-\max \left(\lambda_{i}\right)\right) \max \left(\lambda_{i}\right)} \\
& =K_{\mathrm{re}} \cdot \sqrt{\frac{1}{l-1}} \cdot\left(1-r_{f}\right) \cdot(l-2)
\end{aligned}
$$

where $K_{\text {re }}$ denotes the setting coefficient, which is set to be 1.4. The similarity $r_{i}$ of the two sound feeders is about 1.0. The similarity of one sound feeder and the faulted feeder is denoted by $r_{f,}$. Hence, (18) can be simplified to,

$$
\begin{aligned}
& \lambda_{i}=\sum_{j=1, j \neq i}^{l}\left|\bar{r}_{i}-\bar{r}_{j}\right|=\left(1-r_{f}\right) \cdot \frac{l-2}{l-1} \\
& \lambda_{n}=\sum_{j=1, j \neq n}^{l}\left|\bar{r}_{n}-\bar{r}_{j}\right|=\left(1-r_{f}\right) \cdot(l-2)
\end{aligned}
$$

The results of faulted feeder identification with different numbers of feeders are given in Table VIII. It shows that as the number of feeders increases from 3 to 6 , the sensitivity obtained with the proposed method increases from 1.01 to 1.6.

It should be noted that the proposed method is applicable to the system with three or more feeders in service so that the ATM can be correctly formulated for the implementation of a GRA algorithm.

To further highlight the effectiveness of the proposed method, a comparison with some typical faulted feeder identification methods is provided in Table IX. A weak transient current signal of SPG fault is selected for method verification. A fault occurred at the end of Feeder 3 with $R_{f}=$ $5000 \Omega$ and the fault inception angle is $0^{\circ}$. In addition, white Gaussian noise with a signal-to-noise ratio of $25 \mathrm{~dB}$ is added to emulate harsh conditions. From Table IX, it is clearly observed that the proposed method can identify the faulted feeder accurately. In contrast, incorrect identification results were obtained by the traditional methods such as VCASC, TGRA, and VCFH, as they are less sensitive to weak fault-generated transient signals. Furthermore, the WLPC method failed to identify the faulted feeder under this fault condition.

\section{CONCLUSION}

A novel and simple-to-implement faulted feeder identification method is proposed and discussed in this paper. The adjustment trajectories are obtained by consecutively tuning the arc suppression coil (ASC) around the full compensation state. The trajectories of zero-sequence currents at several steady-state stages are obtained to formulate an adjustment trajectory matrix. Since the similarity of the adjustment trajectories between the faulted line and one of the sound lines is significantly different from that between the two sound lines, the faulted feeder can be identified with high precision. The ASC can be readily controlled to repeatedly generate the adjustment trajectories for further enhancement of the identification reliability. The effectiveness and robustness of the proposed method are also validated. The results show 
that the faulted feeder can be correctly identified with high reliability and robustness in spite of the presence of random noises, different compensation degrees, different adjustment scheme of ASC, different number of feeders, etc. Meanwhile, the results demonstrate that Deng's grey relational analysis is more suitable to obtain the similarity compared to the existing methods.

\section{REFERENCES}

[1] W. Huang and R. Kaczmarek, "Equivalent circuits for an SLG fault distance evaluation by curve fitting in compensated distribution systems," IEEE Trans. Power Del., vol. 23, no. 2, pp. 601-608, Apr. 2008.

[2] IEEE Guide for the Application of Neutral Grounding in Electrical Utility Systems--Part IV: Distribution, IEEE Standard C62.92.4-2014, 2015.

[3] Y. Wang, J. Zhou, Z. Li, Z. Dong, and Y. Xu, "Discriminant-analysis-based single-phase earth fault protection using improved PCA in distribution systems," IEEE Trans. Power Del., vol. 30 , no. 4, pp. 1974-1982, Aug. 2015.

[4] M. Brenna, E. Berardinis, L. Carpini, P. Paulon, P. Petroni, G. Sapienza, G. Scrosati, and D. Zaninelli, "Petersen coil regulators analysis using a real-time digital simulator," IEEE Trans. Power Del., vol. 26, no. 3, pp. 1479-1488, Jul. 2011.

[5] N. Elkalashy, A. Elhaffar, T. Kawady, N. Tarhuni, and M. Lehtonen, "Bayesian selectivity technique for earth fault protection in medium-voltage networks," IEEE Trans. Power Del., vol. 25, no. 4, pp. 2234-2245, Oct. 2010.

[6] D. Gadanayak and R. Mallick, "Interharmonics based high impedance fault detection in distribution systems using maximum overlap wavelet packet transform and a modified empirical mode decomposition," Int. J. Elect. Power Energy Syst., vol. 112, pp. 282-293, Nov. 2019.

[7] M. Barik, A. Gargoom, M. Mahmud, M. Haque, H. Al-Khalidi, and A. Than Oo, "A decentralized fault detection technique for detecting single phase to ground faults in power distribution systems with resonant grounding," IEEE Trans. Power Del., vol. 33, no. 5, pp. 2462-2473, Oct. 2018.

[8] Y. Xue, X. Chen, H. Song, and B. Xu, "Resonance analysis and faulty feeder identification of high-impedance faults in a resonant grounding system," IEEE Trans. Power Del., vol. 32, no. 3, pp. 1545-1555, Jun. 2017.

[9] S. Zhang, Z. He, and R. Mai, "Single-phase-to-ground fault feeder identification based on the feature between voltage and integration of current," IEEJ Trans. Electr. Electron. Eng., vol. 12, no. 5, pp. 683-691, Sep. 2017.

[10] M. Guo and N. Yang, "Features-clustering-based earth fault detection using singular-value decomposition and fuzzy c-means in resonant grounding distribution systems," Int. J. Elect. Power Energy Syst., vol. 93 , pp. 97-108, Dec. 2017.

[11] A. Gharderi, H. A. Mohammadpour, H. L. Ginn, and Y. Shin, "High-impedance fault detection in the distribution network using the time-frequency-based algorithm," IEEE Trans. Power Del., vol. 30, no. 3, pp. 1260-1268, Jun. 2015.

[12] Y. Du, Y. Liu, Q. Shao, L. Luo, J. Dai, G. Sheng, and X. Jiang, "Single line-to-ground faulted line detection of distribution systems with resonant grounding based on feature fusion framework," IEEE Trans. Power Del., vol. 34, no. 4, pp. 1766-1775, Aug. 2019.

[13] W. Wang, K. Zhu, P. Zhang, and W. Xu, "Identification of the faulted distribution line using thyristor-controlled grounding," IEEE Trans. Power Del., vol. 24, no. 1, pp. 52-60, Jan. 2009.

[14] K. Zhu, P. Zhang, W. Wang, and W. Xu, "Controlled closing of PT delta winding for identifying faulted lines," IEEE Trans. Power Del., vol. 26, no. 1, pp. 79-86, Jan. 2011.

[15] X. Lin, J. Huang, and S. Ke, "Faulty feeder detection and fault self-extinguishing by adaptive Petersen coil control," IEEE Trans. Power Del., vol. 26, no. 2, pp. 1290-1291, Apr. 2011.

[16] B. Liu, H. Ma, H. Xu, and P. Ju, "Single-phase-to-ground fault detection with distributed parameters analysis in non-direct grounded systems," CSEE J. Power Energy Syst., vol. 5, no. 1, pp. 139-147, Mar. 2019.

[17] C. Huang, T. Tang, Y. Jiang, L. Hua, and C. Hong, "Faulty feeder detection by adjusting the compensation degree of arc-suppression coil for distribution network," IET Gen. Transmiss. Distrib., vol. 12, no. 4, pp. $807-814,2018$.

[18] P. Wang, B. Chen, H. Zhou, C. Tian, and B. Sun, "Fault location in resonant grounded network by adaptive control of neutral-to-earth complex impedance," IEEE Trans. Power Del., vol. 33, no. 2, pp. 689-698, Apr. 2018.

[19] J. Tang, C. Yang, and L. Cheng, "Analysis on zero-sequence current variation characteristic for feeders of distribution network at different residual current compensation factors," Autom. Electr. Power Syst., vol. 41, no. 13, pp. 125-132, Jul. 2017, (in Chinese).

[20] H. Weng, S. Wang, X. Lin, Z. Li, and J. Huang, "A novel criterion applicable to transformer differential protection based on waveform sinusoidal similarity identification," Int. J. Elect. Power Energy Syst., vol. 105, pp. 305-314, Feb. 2019.

[21] L. Chen, X. Lin, Z. Li, F. Wei, H. Zhao, Z. Bo, J. Huang, and K. Deng, "Similarity comparison based high-speed pilot protection for transmission line," IEEE Trans. Power Del., vol. 33, no. 2, pp. 938-948, Apr. 2018.

[22] Y. Wang, Y. Huang, X. Zeng, G. Wei, J. Zhou, T. Fang, and H. Chen, "Faulty feeder detection of single phase-earth fault using grey relation degree in resonant grounding system," IEEE Trans. Power Del., vol. 32, no. 1, pp. 55-61, Feb. 2017.

[23] X. Li, Z. Wang, L. Zhang, C. Zhou, and D. Dorrell, "State-of-health estimation for Li-ion batteries by combing the incremental capacity analysis method with grey relational analysis," J. Power Sources, vol. 410-411, pp. 106-114, Jan. 2019.

[24] M. Yazdani, C. Kahraman, P. Zarate, and S. Onar, "A fuzzy multi attribute decision framework with integration of QFD and grey relational analysis," Expert Syst. Appl., vol. 115, pp. 474-485, 2019.

[25] J. Tang, B. Xiong, C. Yang, C. Tang, Y. Li, G. Su, and X. Bian, "Development of an integrated power distribution system laboratory platform using modular miniature physical elements: A case study of fault location," Energies, vol. 12, pp. 3780, Oct. 2019.

[26] W. Wang, L. Yan, X. Zeng, B. Fan, and J. M. Guerrero, "Principle and design of a single-phase inverter-based grounding system for neutral-to-ground voltage compensation in distribution networks," IEEE Trans. Ind. Electron., vol. 64, no. 6, pp. 4561-4570, Jun. 2017.

[27] P. Wang, B. Chen, C. Tian, B. Sun, M. Zhou, and J. Yuan, "A novel neutral electromagnetic hybrid flexible grounding method in distribution networks," IEEE Trans. Power Del., vol. 32, no. 3, pp. 1350-1358, Jun. 2017.

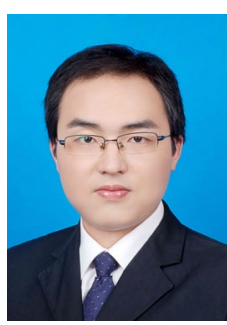

Jinrui Tang (M'16) received the B.S. degree in electrical engineering from Zhejiang University, Hangzhou, China, in 2009, and the Ph.D. degree in electrical engineering from the School of Electrical and Electronics Engineering, Huazhong University of Science and Technology, Wuhan, China, in 2014. He is currently an Associate Professor with the School of Automation, Wuhan University of Technology, Wuhan. His research interests include power system protection.

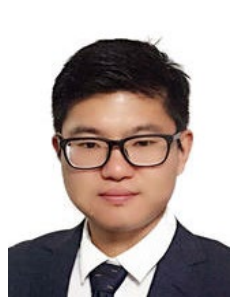

Binyu Xiong (S'11-M'16) received the B.E. degree in electrical engineering from Wuhan University, Wuhan, China, in 2010, and the MSc. and the Ph.D. degrees from Nanyang Technological University, Singapore in 2011 and 2016 respectively, both in power engineering. He is currently an Associate Professor with the School of Automation, Wuhan University of Technology, Wuhan. His research interests include battery modeling, state of charge estimation, large-scale energy storage systems, power electronics, and renewable energy generations. 


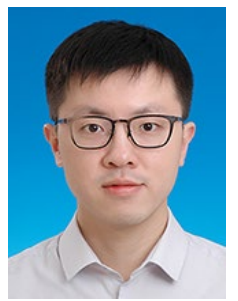

Yang Li (S'11-M'16) received the B.E. degree in electrical engineering from Wuhan University, Wuhan, China, in 2007 and the Ph.D. degree in power engineering from Nanyang Technological University (NTU), Singapore, in 2015. From 2015 to 2018, he was a Research Fellow in the Energy Research Institute, NTU, and the School of Electrical Engineering and Computer Science, Queensland University of Technology, Brisbane, Australia, respectively. Since 2019, he has been with the School of Automation, Wuhan University of Technology, Wuhan. His research interests include modeling and control of renewable generation systems, energy storage systems, and power electronic converters.

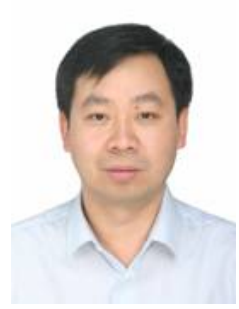

Chengqing Yuan, received the B.S. degree in chemical engineering from Wuhan Automotive Polytechnic University, Wuhan, China, in 1998, the M.Sc. degree in mechanical design and theory from Wuhan Research Institute of Materials Protection, Wuhan, in 2001, and the $\mathrm{Ph} . \mathrm{D}$. degree in vehicle operation engineering from the School of Energy and Power Engineering, Wuhan University of Technology, Wuhan, in 2005, where he is currently a Professor. His research is focused on marine new energy, tribology, and condition monitoring.

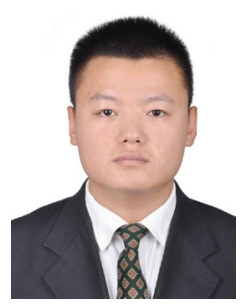

Yuanchao Qiu received the B.S. and the M.S. degrees in vehicle operation engineering from the School of Energy and Power Engineering, Wuhan University of Technology, Wuhan, China, in 2014 and 2016, respectively, where he is currently working toward the $\mathrm{Ph} . \mathrm{D}$ degree in vehicle operation engineering. His main research interest is focused on the power quality in the green ship and energy storage system optimization. 